\title{
Lymphangioleiomyomatosis Unusual Cause of Spontaneous Pneumothorax
}

\author{
Lamya Al Aamri ${ }^{1}$, Raafat Dahrouj ${ }^{1}$, Nasser Al Awaid ${ }^{2}$, Rubyath Rajib ${ }^{3}$ \\ ${ }^{1}$ Internal Medicine Specialist, Sultan Qaboos Hospital, Salalah, Oman. \\ ${ }^{2}$ Consultant Pulmonologist, Head of Department of Medicine, Sultan Qaboos Hospital, Salalah. \\ ${ }^{3}$ Senior Consultant, Histopathologist, Sultan Qaboos Hospital, Salalah.
}

Corresponding Author: Lamya Al Aamri

\begin{abstract}
Lymphangioleiomyomatosis (LAM) is a rare multisystem disease, predominately affect premenopausal female. LAM could be an inherited disease associated with Tuberous Sclerosis Complex syndrome or sporadic. Most common pulmonary symptoms are dyspnea and pneumothorax. We report 31- year female, presented with right-side pneumothorax chest drainage was inserted. Further investigation revealed multiple cystic lesions in chest computed tomography images suggestive of LAM disease. She underwent video-assisted thoracoscopic surgery (VATs) to obtain a lung biopsy. Histopathology lung tissue confirms pulmonary Lymphangioleiomyomatosis.
\end{abstract}

Keywords: Lymphangioleiomyomatosis; Tuberous Sclerosis Complex syndrome; video-assisted thoracoscopic surgery

\section{INTRODUCTION}

Spontaneous pneumothorax can result in many lung diseases condition, Lymphangioleiomyomatosis (LAM) is unusual cause. LAM is a rare multisystem disease with clinical manifestations pulmonary and extrapulmonary; it almost occurs exclusively in premenopausal women. ${ }^{1,2}$ LAM may coexist with Tuberous Sclerosis Complex syndrome (TSC-LAM) patients or sporadic (LAMs). ${ }^{3}$

Here we report 31 years Omani female presented with pneumothorax related to Lymphangioleiomyomatosis.

\section{CASE PRESENTATION}

A 31-year- old lady was unmarried admitted to Sultan Qaboos hospital, Salalah, with a chief complaint of chest pain for 3 days. She had right side chest pain, which started suddenly and worsening over time, along she had dysmenorrhea. She denied shortness of breath, cough, or hemoptysis. Her medical history included post laparotomy myomectomy. On physical examination revealed no skin lesion or eye problem or features of marfonid. Her vitals were stable, no trachea deviation, chest reduces breath sound on the right side.

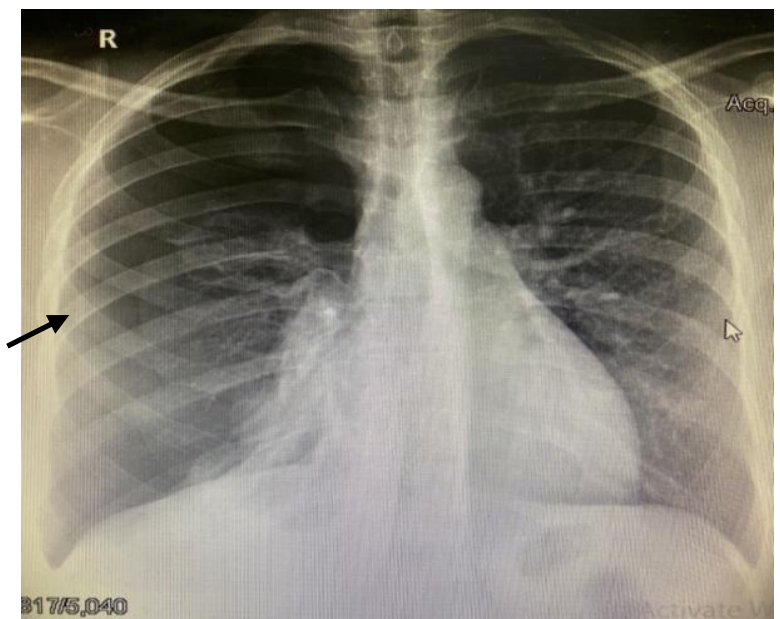

Figure 1: Chest $\mathrm{x}$-ray image showed right sided pneumothorax (arrow)

She was investigated with a chest $\mathrm{x}$ ray that showed right-side pneumothorax (Figure 1). Subsequently, an intercostal 
drainage tube was inserted in the right- side thorax. Due to the relation timing of pneumothorax with her menstruation rising possibilities of catamenial pneumothorax, A high-resolution Computed Tomography scan was ordered. It revealed multiple small thin-walled cystic lesions 2.5 to $5 \mathrm{~mm}$ scattered predominantly in upper and mid zones likely to be lymphangioleiomyomatosis LAM (Figure 2).

Further evaluation using ultrasound abdomen revealed no shreds of evidence of renal angiomyolipoma or abdomen lymphangioleiomyomas. Unfortunately, Serum VEGF-D was unavailable in our center. Eventually, pneumothorax resolved and chest tube drainage was removed.

To confirm a diagnosis of lymphangioleiomyomatosis LAM lung biopsy was Obtain by video-assisted thoracoscopic surgery (VATs).
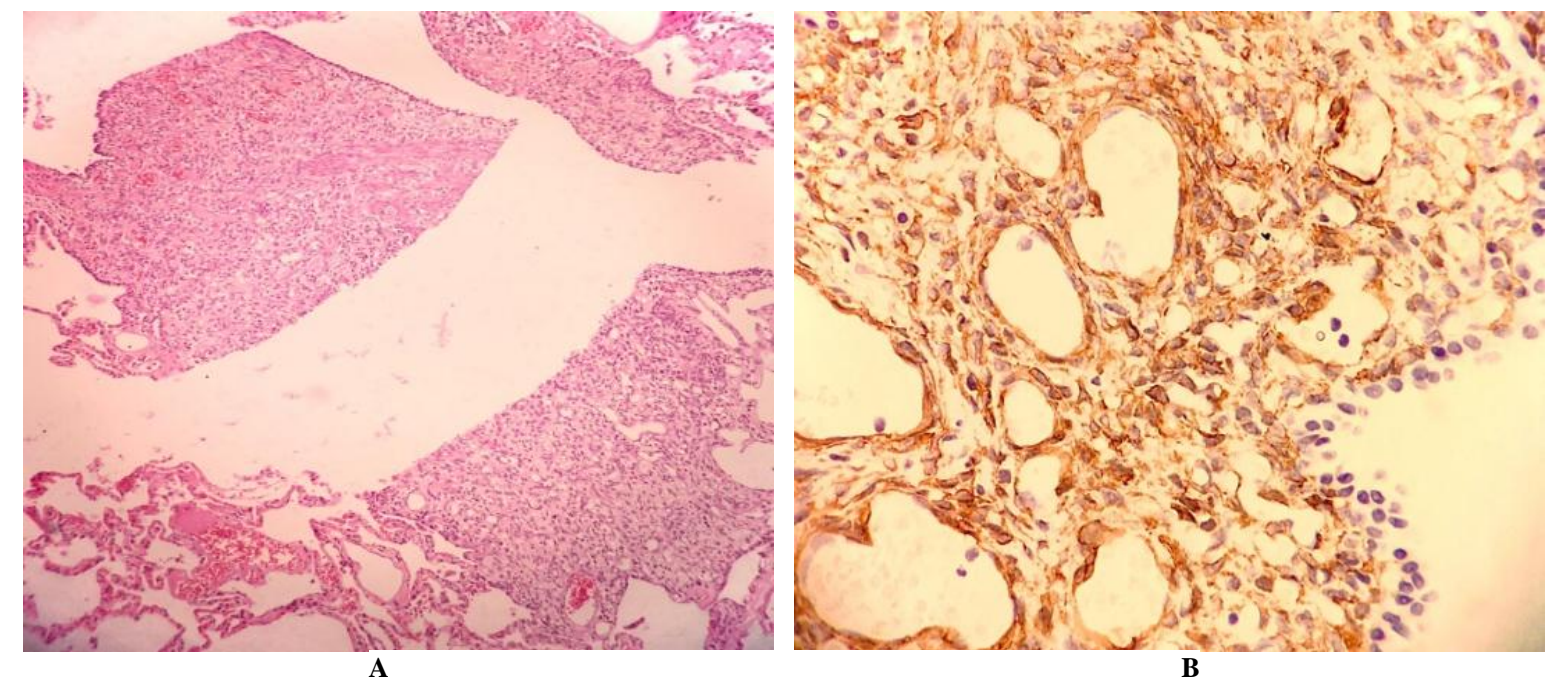

Figure 3: Histopathology: foci of spindle cells admixed with capillaries, H\&E, x10 (A). Spindle cells, positive for smooth muscle (SMA) immune-stain, $\mathbf{x 4 0}$ (B).

She had been seen in the chest clinic, a repeated chest $\mathrm{x}$-ray showed no recurrence and spirometry value of (FEV1: 2.38, FVC:2.79, FEV1/FVC: 85).

\section{DISCUSSION}

Pulmonary

Lymphangioleiomyomatosis is an air-filled cystic lung disease distinctive by smooth muscle proliferation (LAM cells) lining airway, lymphatics and vassals lead to
Histopathology finding irregular vascular space surrounded by bundles of spindle cells. Which were positive for SMA (smooth muscle antigen), estrogen and progesterone receptors. The morphological appearances confirm LAM (Figure 3).

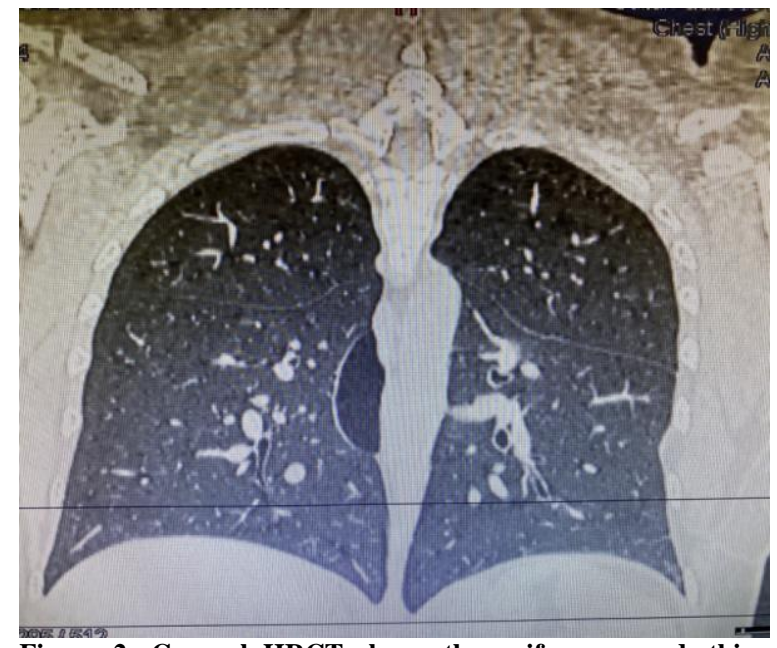

Figure 2: Coronal HRCT shows the uniform, round, thinwalled cysts. 
activation of $\mathrm{mTOR}$ result in proliferation of abnormal smooth muscle cells. ${ }^{4}$

The prevalence of LAM in female 1: $1,000,000$ sporadic and $30-40 \%$ of TSC patients have LAM. ${ }^{1,3}$

Theories suggest estrogen contribution as result of sporadic LAM exclusive found in young women.3 However, men with TSC developed LAM disease. $^{5}$

There are no specific pulmonary symptoms however most common dyspnea and pneumothorax. ${ }^{6}$ other symptoms are wheeze, hemoptysis, and chylous effusions.6

Forty to fifty percent of the LAM population developed spontaneous pneumothorax as the first symptoms. ${ }^{7}$ Approximately, $73-85 \%$ of LAM patients have a recurrence a pneumothorax with $71 \%$ ipsilateral. $^{7,8}$

Factors that increased the risk of pneumothorax in LAM patients include Smoking, pregnancy, and larger cyst size on HRCT (>5mm).7

Moreover, LAM patients presenting with pneumothorax have a good prognosis compared to those presenting with dyspnea. ${ }^{9}$

In our case, the patient is young woman who presented with pneumothorax as the first clinical symptom and denied any history of shortness of breath, with no other clinical manifestation of TSC or extrapulmonary features.

The pulmonary function can be used to assess the severity of LAM disease and may be revealed a normal to an obstructed pattern. Also, may show a decline in the $\mathrm{DL}_{\mathrm{CO}}$, and impaired gas exchange. ${ }^{910}$

Computed tomography serving as diagnostic tool in addition to assessing lung disease severity. Studies showed by evaluating the percentage of cysts volumes involved in lung parenchyma, it correlated with $\mathrm{FEV}_{1}$, residual volume, and $\mathrm{DL}_{\mathrm{CO}}{ }^{10}$

The European Respiratory Society guides diagnosis of LAM disease need evidence of cystic lung disease plus other features; tuberous sclerosis, renal angiomyolipoma, chylous, lymphangioleiomyoma, or high serum VEGF-D above or equal $800 \mathrm{pg} / \mathrm{ml} .1$

Lung biopsy need when the presence of cystic lung disease with a lack of others features. 1

Lung biopsy Histopathology confirmation presence of LAM cells composed from two cells small spindleshaped and larger epithelioid cells. The epithelioid cells usually react with HMB-45 compared to spindle cells react to PCNA is classic confirmation, although it is not necessary for diagnosis. Receptors for estrogen and progesterone have been reacting to LAM cells. 1

In our patient, HRCT was done in the first presentation allowed the early discovery of mild LAM disease. Further confirmation is needed by lung biopsy via VAST due to lack of extrapulmonary features and unavailability of VEGF-D test.

In our case, lung biopsy histopathology confirmed the presence of cystic space as well as a proliferation of spindle cells which one required for the diagnosis of LAM. Those spindle cells were immune-histologically positive for estrogen receptor, progesterone receptor, and smooth antigen. Although they are usually reactive for HMB-45, it is not mandatory for diagnosis.

Spontaneous pneumothorax should treat with tube thoracostomy. ${ }^{711}$ Patients treated conservatively have a high chance of recurrence approximately $66 \%$ compared with chemical or surgical pleurodesis approaches $\left(27 \%, 32 \%\right.$ respectively). ${ }^{7,11}$

Sirolimus and everolimus are Mammalian targets of rapamycin (mTOR) inhibitors revealed effectiveness in treating extrapulmonary. ${ }^{.12}$ It may benefit in improving mild to moderate FEV.12

Progressive LAM disease with oxygen-dependent patients or where $\mathrm{FEV}_{1}$ is less than $30 \%$ predicted consider lung transplantation.

Our patient underwent an intercostal draining tube with completed resolving pneumothorax with no recurrence. Spirometry was normal and Follow up 
images showed mild LAM with no change, for this patient recommendation for monitoring.

\section{CONCLUSION}

Lymphangioleiomyomatosis should be suspect in young females presented with spontaneous pneumothorax. HRCT is critical for recognized disease and early treatment.

\section{Acknowledgement: None}

\section{Conflict of Interest: None}

\section{Source of Funding: None}

\section{REFERENCES}

1. S.R. Johnson, J.F. Cordier, R. Lazor, V. Cottin, U. Costabel, S. Harari, et al. Kingswood. European Respiratory Society guidelines for the diagnosis and management of lymphangioleiomyomatosis. Eur Respir J. 2010 Jan;35(1):1426; DOI: $10.1183 / 09031936.00076209$

2. M.K. Glassberg. Lymphangioleiomyomatosis. Clin Chest Med. 2004 Sep;25(3):573-82, vii. doi: 10.1016/j.ccm.2004.05.003.

3. S.R.

Johnson. Lymphangioleiomyomatosis. Eur Respir J. 2006 May;27(5):1056-65. doi: 10.1183/09031936.06.00113303.

4. J. Moss, N.A. Avila, P.M. Barnes, R.A. Litzenberger, J. Bechtle, P.G. Brooks, et al. Prevalence and clinical characteristics of lymphangioleiomyomatosis (LAM) in patients with tuberous sclerosis complex. Am J Respir Crit Care Med. 2001;164(4):669-71. doi: 10.1164/ajrccm.164.4.2101154

5. M.C. Aubry, J.L. Myers, J.H. Ryu, E.P. Henske, H. Logginidou, S.M. Jalal, H.D. Tazelaar. Pulmonary lymphangioleiomyomatosis in a man. Am J Respir Crit Care Med. 2000 Aug; 162(2 Pt 1):749-52. doi: 10.1164/ajrccm.162.2.9911006.
6. J.H. Ryu, J. moss, G.J. Beck, J.C. Lee, K.K. Brown, et al. The NHLBI Lymphangioleiomyomatosis Registry Characteristics of 230 Patients at Enrollment. Am J Respir Crit Care Med. 2006 Jan 1; 173(1): 105-111. doi.org/10.1164/rccm.200409$12980 \mathrm{C}$

7. J. Cooley, Y.C. Gary Lee, N. Gupta. Spontaneous Pneumothorax in Diffuse Cystic Lung Diseases. Curr Opin Pulm Med. 2017 Jul; 23(4): 323-333. doi: 10.1097/MCP.0000000000000391.

8. W.K. Steagall, C.G. Glasgow, O.M. Hathaway, N.A. Avila, A.M. TaveiraDasilva, A. Rabel, et al. Genetic and morphologic determinants of pneumothorax in lymphangioleiomyomatosis. Am J Physiol Lung Cell Mol Physiol. 2007;293(3):L800-8. doi: 10.1152/ajplung.00176.2007.Epub 2007 Jul

9. M. Hayashida, K. Seyama, Y. Inoue, et al. The epidemiology of lymphangioleiomyomatosis in Japan: A nationwide cross-sectional study of presenting features and prognostic. factors. Respirology. 2007; 12:523-530. doi: 10.1111/j.1440-1843.2007.01101.x.

10. A.M. Taveira- DaSilva, G. PachecoRodriguez, J. Moss. The Natural History of Lymphangioleiomyomatosis: Markers of Severity, Rate of Progression and Prognosis. Lymphat Res Biol. 2010 Mar;8(1):9doi: $10.1089 / \mathrm{lrb} .2009 .0024$

11. K.F. Almoosa, J.H. Ryu, J. Mendez, J.T. Huggins, L.R. Young, E.J. Sullivan, et al. Management of pneumothorax in lymphangioleiomyomatosis: effects on recurrence and lung transplantation complications. Chest. 2006;129(5):1274 -81. doi: 10.1378/chest.129.5.1274.

12. C.S. Goncalves Freitas, B.G. Baldi, M.S. Araújo. Use of sirolimus in the treatment of lymphangioleiomyomatosis: favorable responses in patients with different extrapulmonary manifestations. J Bars 
Pneumol. 2015 May-Jun; 41(3): 275280. DOI: $10.1590 / \mathrm{S} 1806-$

How to cite this article: Lamya Al Aamri, 37132015000004553

Dahrouj R, Nasser Al Awaid et.al. 13. M.M. Cohen, S. Pollock-BarZiv, S.R. Johnson. Emerging clinical picture of lymphangioleiomyomatosis. Thorax. 20 05;60:875-879. doi: Lymphangioleiomyomatosis unusual cause of spontaneous pneumothorax. Int $J$ Health 10.1136/thx.2004.035154 Sci Res. 2021; 11(6): 226-230. DOI: https://doi.org/10.52403/ijhsr.20210635 